\title{
Dual-source computed tomography of the lung with spectral shaping and advanced iterative reconstruction: potential for maximum radiation dose reduction
}

\author{
Matthias Wetzl ${ }^{1}$ D $\cdot$ Matthias S. May ${ }^{1,2} \cdot$ Daniel Weinmann $^{1} \cdot$ Matthias Hammon $^{1} \cdot$ Christoph Treutlein $^{1} \cdot$ \\ Martin Zeilinger ${ }^{1} \cdot$ Alexander Kiefer $^{3} \cdot$ Regina Trollmann $^{3} \cdot$ Joachim Woelfle $^{3} \cdot$ Michael Uder $^{1,2} \cdot$ Oliver Rompel $^{1}$
}

Received: 2 October 2019 / Revised: 6 March 2020 / Accepted: 12 May 2020 / Published online: 17 June 2020

(C) The Author(s) 2020

\begin{abstract}
Background Radiation dose at CT should be as low as possible without compromising diagnostic quality.

Objective To assess the potential for maximum dose reduction of pediatric lung dual-source CT with spectral shaping and advanced iterative reconstruction (ADMIRE).

Materials and methods We retrospectively analyzed dual-source CT acquisitions in a full-dose group (FD: $100 \mathrm{kV}, 64$ reference $\mathrm{mAs}$ ) and in three groups with spectral shaping and differing reference mAs values (Sn: $100 \mathrm{kV}, 96 / 64 / 32 \mathrm{reference} \mathrm{mAs),} \mathrm{each}$ group consisting of 16 patients (age mean 11.5 years, standard deviation 4.8 years, median 12.8 years, range 1.3-18 years). Advanced iterative reconstruction of images was performed with different strengths (FD: ADMIRE Level 2; Sn: ADMIRE Levels 2, 3 and 4). We analyzed dose parameters and measured noise. Diagnostic confidence and detectability of lung lesions as well as anatomical structures were assessed using a Likert scale (from 1 [unacceptable] to 4 [fully acceptable]).

Results Compared to full dose, effective dose was reduced to $16.7 \%$ in the Sn 96 group, 11.1\% in Sn64, and 5.5\% in Sn32 $(P<0.001)$. Noise values of Sn64 $\mathrm{ADM}_{4}$ did not statistically differ from those in $\mathrm{FD}_{\mathrm{ADM} 2}(45.7$ vs. 38.9 Hounsfield units [HU]; $P=0.132)$, whereas noise was significantly higher in $\mathrm{Sn} 32_{\mathrm{ADM} 4}$ compared to $\operatorname{Sn}_{64}{ }_{\mathrm{ADM} 4}(61.5 \mathrm{HU} ; P<0.001)$. A Likert score $>3$ was reached in $\mathrm{Sn}_{6} 4_{\mathrm{ADM} 4}$ regarding diagnostic confidence (3.2) and detectability of lung lesions (3.3). For detectability of most anatomical structures, no significant differences were found between $\mathrm{FD}_{\mathrm{AM} 2}$ and $\operatorname{Sn} 64_{\mathrm{ADM} 4}(P \geq 0.05)$.

Conclusion In pediatric lung dual-source CT, spectral shaping together with ADMIRE 4 enable radiation dose reduction to about $10 \%$ of a full-dose protocol while maintaining an acceptable diagnostic quality.
\end{abstract}

Keywords Adolescents · Advanced iterative reconstruction · Children · Dual-source computed tomography · Tin prefiltration · Lung $\cdot$ Radiation dose reduction $\cdot$ Spectral shaping

Matthias Wetzl

Matthias.wetzl@uk-erlangen.de

1 Department of Radiology, University Hospital Erlangen, Erlangen, Germany

2 Imaging Science Institute, University Hospital Erlangen, Erlangen, Germany

3 Department of Pediatrics and Adolescent Medicine, University Hospital Erlangen, Erlangen, Germany

\section{Introduction}

Dual-source CT is widely used for evaluating lung diseases in children and adolescents. In general, different approaches to decreasing $\mathrm{CT}$ radiation exposure have been proposed. One of the most effective methods is the reduction of tube voltage, because the dose increases with the square of the tube voltage. On the other hand, it varies approximately linearly with tube current [1].

Recently, third-generation dual-source CT scanners have been equipped with additional tin prefiltration that removes low-energy photons of the X-ray beam. These photons contribute little to image quality but increase radiation burden. The so-called spectral shaping has enabled radiation dose 
reduction in several anatomical regions in adults and children [2-5].

It has been reported that advanced iterative reconstruction enables reduction of radiation dose while preserving image quality in pediatric CT examinations [6]. Newell et al. [7] reported a phantom study indicating that third-generation dual-source CT scanners using third-generation iterative reconstruction methods (ADMIRE; Siemens Healthcare, Erlangen, Germany) can generate accurate quantitative CT images with acceptable image noise at very low dose levels. In a study of Rompel et al. [8], chest CT angiography in newborns and young children performed with a third-generation dual-source CT scanner using a $70-\mathrm{kV}$ protocol together with stronger reconstruction levels of ADMIRE allowed high image quality at low radiation dose level.

We hypothesized that pediatric lung dual-source CT spectral shaping together with a strong reconstruction increment of ADMIRE would enable substantial radiation dose reduction while maintaining an acceptable diagnostic quality. Accordingly, the aim of this study was to identify the percentage value of possible dose reduction compared to a full-dose examination protocol.

\section{Materials and methods}

We conducted this study in accordance with the guidelines of the Declaration of Helsinki; our local ethics committee approved the study. Written informed consent for dual-source CT of the lung was obtained for all patients. The institutional review board waived supplemental agreement because of the retrospective study design.

\section{Patient characteristics}

A total of 64 patients with dual-source CT examinations of the lung were enrolled in this study. They were retrospectively selected from four examination protocols available in our department. In 16 patients (age $11.2 \pm 5.0$ years, median 12.4 years, range 2.9-17.7 years) a full-dose (FD) dualsource CT of the lung had been conducted. Forty-eight other patients had been examined using one of three reduced-dose protocols with tin prefiltration $(\mathrm{Sn})$ established in our department (Sn96: $n=16,10.3 \pm 6.1$ years, median 11.6 years, range 1.3-17.7 years; Sn64: $n=16,13.1 \pm 3.4$ years, median 13.1 years, range $5.6-18.0$ years; $S n 32: n=16,11.4 \pm 4.2$ years, median 12.8 years, range $4.8-17.6$ years). The $\mathrm{Sn}$ protocols had been implemented at our institute in order to gradually reduce radiation exposure in clinical routine. Patients of the different groups were matched for age, weight and body mass index. Among all groups there was no significant difference in patient characteristics (Table 1).
All patients had been referred for CT to further investigate suspected or known non-cancer lung diseases such as cystic fibrosis, primary ciliary dyskinesia, prolonged course of pneumonia, chronic lung complications of pneumonia, suspected pulmonary hemorrhage, aspiration pneumonitis, pulmonary Langerhans cell histiocytosis, tuberculosis, and atelectasis or pleural effusion of unclear origin.

\section{Dual-source computed tomography techniques}

All dual-source CT examinations were performed using the same third-generation scanner (Somatom Definition Force, Siemens Healthcare). CT parameters were as follows: $0.25 \mathrm{~s}$ gantry rotation time, detector collimation of $2 \times 96 \times 0.6 \mathrm{~mm}$, slice collimation of $192 \times 0.6 \mathrm{~mm}$ using $\mathrm{z}$-flying focal spot technique, spiral pitch factor 3.0, tube voltage modulation switched off. In the full-dose protocol, patients were examined at a $100-\mathrm{kV}$ setting with automatic exposure control (reference tube current time product per rotation $64 \mathrm{mAs}$; CareDose4D, Siemens Healthcare). In all other protocols 0.6-mm tin prefiltration was applied. Because tin prefiltration is only available at $100-\mathrm{kV}$ and $150-\mathrm{kV}$ tube voltage, with higher diagnostic dose efficiency at $100 \mathrm{kV}$ [9], the lower $\mathrm{kV}$ setting is used in our department. For the three examination protocols with spectral shaping, default values of reference tube current-time product per rotation were $96 \mathrm{mAs} / 64 \mathrm{mAs} / 32$ mAs. Examinations were performed in supine position with elevated arms from the upper to the lower thoracic aperture. If necessary, a body-weight-adapted dose of iodinated contrast medium was injected intravenously (iomeprol $300 \mathrm{mg} / \mathrm{mL}$, Iomeron, Bracco Imaging, Konstanz, Germany; or Accutron CT-D, Medtron AG, Saarbrücken, Germany).

\section{Postprocessing}

Primary image data were automatically generated with a slice thickness of $0.6 \mathrm{~mm}$ using filtered back-projection (FBP). Additionally, all data sets of examination protocols including tin prefiltration were generated with advanced iterative reconstruction utilizing a medium, an intermediate and a strong increment (ADMIRE strengths 2/3/4). Slice thickness was $0.6 \mathrm{~mm}$, in these protocols, too. In our clinical practice we observed adequate diagnostic quality on full-dose examinations when ADMIRE 2 was used. Consequently, reconstruction of ADMIRE 3 and ADMIRE 4 had not been performed at the time of examinations and thus was not available in the retrospective setting of this study. Iterative reconstruction is characterized by repeated forward and back projection of raw data and image data in combination with statistical modeling. The repeated comparison of projected raw data with the measured data allows removal of geometric imperfections. ADMIRE is built upon these principles, with substantial modifications, allowing a high iteration speed [7]. It has been 
Table 1 Patient characteristics of the different dose groups

\begin{tabular}{llllll}
\hline Dose group & Full-dose & Sn96 & Sn64 & Sn32 & $P$-value $^{\mathrm{a}}$ \\
\hline Number of patients & 16 & 16 & 16 & 16 & \\
Gender & 9 male, & 9 male, & 11 male, & 12 male, & \\
& 7 female & 7 female & 5 female & 4 female & \\
Age: mean \pm SD, & $11.2 \pm 5.0$ & $10.3 \pm 6.1$ & $13.1 \pm 3.4$ & $11.4 \pm 4.2$ & ANOVA, \\
$\quad$ median (range), years & 12.4 & 11.6 & 13.1 & 12.8 & $P=0.435$ \\
& $(2.9-17.7)$ & $(1.3-17.7)$ & $(5.6-18.0)$ & $(4.8-17.6)$ & \\
Weight: & $40.9 \pm 22.9$ & $36.1 \pm 19.3$ & $46.9 \pm 16.1$ & $45.6 \pm 24.1$ & ANOVA, \\
$\quad$ & & & & $P=0.457$ \\
mean \pm SD, kg & $17.8 \pm 3.8$ & $18.5 \pm 4.2$ & $18.7 \pm 3.2$ & $19.3 \pm 5.3$ & ANOVA, \\
$\quad$ mean \pm SD & & & & & $P=0.791$ \\
\hline
\end{tabular}

ANOVA analysis of variance, $S D$ standard deviation, $S n 96 / S n 64 / S n 32$ dose groups with tin prefiltration and different reference tube current-time products (96/64/32 reference mAs, respectively)

${ }^{\text {a }} P$-value $<0.05$ is significant shown that ADMIRE has the potential to significantly improve image quality while reducing noise and artifacts in CT scans $[8,10,11]$. In ADMIRE, images are reconstructed by minimizing the objective function incorporated with an accurate system model, a statistical noise model, and a prior model [12].

All images were anonymized and transferred to a postprocessing 3-D console (SyngoVia VA30A; Siemens Healthcare).

\section{Image analysis}

Images were analyzed independently by two radiologists (O.R. and M.H., with 25 years and 10 years of experience in pediatric lung CT, respectively), following the European Guidelines on Quality Criteria for CT. The ratings of the two readers were averaged. For all images, a dedicated lung convolution kernel (B157) was used, as recommended by the manufacturer. Images were interpreted in axial, coronal and sagittal orientation with 1-mm slice thickness using a multiplanar imaging tool (MM Reading, SyngoVia VA30A; Siemens Healthcare). Maximum- and minimum-intensity projections were allowed to be used at the discretion of the readers. The default window setting was center $-600 \mathrm{HU}$ and width 1,700 HU and could be individually adjusted by the readers.

We rated diagnostic confidence as well as detectability of the following anatomical structures on a 4-point Likert scale (1 unacceptable, 2 acceptable under limited conditions, 3 probably acceptable, 4 fully acceptable): medium-size and small pulmonary vessels, tertiary bronchi, lung fissures, lung parenchyma. We also rated suspicious lung lesions with respect to detectability, contrast and contour sharpness using the same 4-point Likert scale.

To assess image quality, we measured noise in the tracheal lumen on 1.0-mm-thick axial images of all datasets (FBP,
ADMIRE 2/3/4). Ten randomly selected patients were evaluated ex ante to detect the optimal surface of the circular region of interest (ROI) with respect to the anatomical target regions. Thus, the defined size of ROI was $0.4 \mathrm{~cm}^{2}$ for older children and adolescents and $0.2 \mathrm{~cm}^{2}$ for smaller children. For each axial image, we performed and averaged three measurements. Image noise was defined as the standard deviation of the attenuation value.

\section{Radiation exposure and effective dose}

Radiation exposure was assessed as volumetric CT dose index $\left(\mathrm{CTDI}_{\mathrm{vol}}\right)$ and dose-length product (DLP). Estimated effective dose (ED) was calculated as DLP·k, using an individual linear interpolation of the conversion factor reported in literature for chest $\mathrm{CT}$ at $100 \mathrm{kV}$ between neonates $\left(\mathrm{k}_{0}=0.0739 \mathrm{mSv} / \mathrm{mGy} \cdot \mathrm{cm}\right), 1$-year-olds $\left(\mathrm{k}_{1}=0.048 \mathrm{mSv} /\right.$ $\mathrm{mGy} \cdot \mathrm{cm})$, 5-year-olds $\left(\mathrm{k}_{5}=0.0322 \mathrm{mSv} / \mathrm{mGy} \cdot \mathrm{cm}\right), 10$-yearolds $\left(\mathrm{k}_{10}=0.0235 \mathrm{mSv} / \mathrm{mGy} \cdot \mathrm{cm}\right)$ and 18 -year-olds $\left(\mathrm{k}_{18}=0.0144 \mathrm{mSv} / \mathrm{mGy} \cdot \mathrm{cm}\right)$ as a function of days of age $[8$, $13]$.

\section{Statistical analysis}

Statistical analysis was performed using SPSS software version 25 (IBM, Armonk, NY) and WINPEPI (Abramson JH, Hebrew University, Jerusalem). Values are given as mean \pm standard deviation if normal distribution was assumed by Kolmogorov-Smirnov tests. Nominal variables were also expressed as frequencies. For multiple comparisons one-way analysis of variance (ANOVA) multiple comparison test with Bonferroni and Games-Howell post hoc pairwise comparisons were applied. All tests were performed two-sided, and $P<0.05$ was considered to be statistically significant. We calculated proportion of inter-rater disagreement and information-based measure of disagreement (IBMD). IBMD 
measures the level of disagreement between two or more observers. A value of 0 indicates no disagreement, whereas a value of 1 indicates total disagreement [14].

\section{Results}

\section{Diagnostic confidence}

Diagnostic confidence improved in all Sn groups with increasing strength levels of ADMIRE (Table 2). There was no significant difference in diagnostic confidence between the fulldose group reconstructed with ADMIRE 2 and the Sn96 group reconstructed with ADMIRE 4 (FD ADM2 $_{2 s}$. Sn96 $\left.{ }_{\mathrm{ADM} 4}: P=0.092\right)$. Although differences between the $\mathrm{FD}_{\mathrm{ADM} 2}$ group and the $\mathrm{Sn} 64_{\mathrm{ADM} 4}$ and $\mathrm{Sn} 32_{\mathrm{ADM}} 4$ groups were significant $\left(\mathrm{FD}_{\mathrm{ADM} 2}\right.$ vs. Sn64 ${ }_{\mathrm{ADM} 4}: P=0.008 ; \mathrm{FD}_{\mathrm{ADM}}$ vs Sn32 ADM $\left._{4}: P<0.001\right)$, diagnostic confidence reached a Likert score $>3$ in the $\mathrm{Sn} 64_{\mathrm{ADM}} 4$ group. This was not true for the $\mathrm{Sn} 32_{\mathrm{ADM}} 4$ group (2.7). For further information see Table 2. The two readers disagreed in 50 of 224 ratings (22\%, IBMD $0.10,95 \%$ confidence interval [CI] 0.08-0.12).

\section{Anatomical structures}

In all Sn groups, detectability of anatomical structures improved with increasing strength levels of ADMIRE (Table 2). Compared to $\mathrm{FD}_{\mathrm{ADM} 2}$, values for $\mathrm{Sn} 96_{\mathrm{ADM} 4}$, Sn64 $4_{\mathrm{ADM} 4}$ and $\mathrm{Sn} 32_{\mathrm{ADM} 4}$ were $3.4 \pm 0.6$ vs. $3.2 \pm 0.6,3.1 \pm 0.4$ and $2.5 \pm 0.6$ for small vessels; $3.8 \pm 0.4$ vs. $3.8 \pm 0.4,3.7 \pm 0.4$ and $3.5 \pm 0.5$ for tertiary bronchi; and $3.5 \pm 0.7$ vs. $2.8 \pm 0.7,3.0$ \pm 0.6 and $2.2 \pm 0.5$ for lung fissures. Except for lung

Table 2 Diagnostic confidence and detectability of anatomical structures of different dose groups

\begin{tabular}{|c|c|c|c|c|c|c|c|}
\hline Dose group & & FD & $\operatorname{Sn} 96$ & Sn64 & $\operatorname{Sn} 32$ & Relevant $P$-values ${ }^{\mathrm{c}}$ & \\
\hline \multirow[t]{4}{*}{ Diagnostic confidence } & FBP & $3.7 \pm 0.4$ & $2.3 \pm 0.6$ & $1.9 \pm 0.6$ & $1.3 \pm 0.3^{\mathrm{a}, \mathrm{b}}$ & $\mathrm{FD}_{\mathrm{ADM} 2}$ vs. $\mathrm{Sn} 64_{\mathrm{ADM} 4}$ & $P=0.008$ \\
\hline & ADMIRE 2 & $3.8 \pm 0.5$ & $2.7 \pm 0.4$ & $2.5 \pm 0.4$ & $1.8 \pm 0.4^{\mathrm{a}, \mathrm{b}}$ & $\mathrm{FD}_{\mathrm{ADM} 2}$ vs. $\mathrm{Sn} 32_{\mathrm{ADM} 4}:$ & $P<0.001$ \\
\hline & ADMIRE 3 & & $3.3 \pm 0.5$ & $2.8 \pm 0.5$ & $2.3 \pm 0.4^{\mathrm{a}, \mathrm{b}}$ & $\mathrm{Sn} 64_{\mathrm{ADM} 4}$ vs. $\mathrm{Sn} 32_{\mathrm{ADM} 4}$ & $P=0.058$ \\
\hline & ADMIRE 4 & & $3.3 \pm 0.5$ & $3.2 \pm 0.4$ & $2.7 \pm 0.6^{\mathrm{a}}$ & & \\
\hline \multirow[t]{4}{*}{ Medium-size vessels } & FBP & $3.8 \pm 0.5$ & $3.6 \pm 0.7$ & $3.3 \pm 0.4$ & $2.4 \pm 0.5^{\mathrm{a}, \mathrm{b}}$ & $\mathrm{FD}_{\mathrm{ADM} 2}$ vs. $\mathrm{Sn} 64_{\mathrm{ADM} 4}:$ & $P=1$ \\
\hline & ADMIRE 2 & $3.8 \pm 0.4$ & $3.7 \pm 0.6$ & $3.6 \pm 0.4$ & $3.0 \pm 0.6^{\mathrm{a}, \mathrm{b}}$ & $\mathrm{FD}_{\mathrm{ADM} 2}$ vs. $\mathrm{Sn} 32_{\mathrm{ADM} 4}:$ & $P=0.036$ \\
\hline & ADMIRE 3 & & $3.8 \pm 0.5$ & $3.8 \pm 0.4$ & $3.1 \pm 0.7^{\mathrm{a}, \mathrm{b}}$ & Sn64 $4_{\mathrm{ADM} 4}$ vs. Sn32 $2_{\mathrm{ADM} 4}:$ & $P=0.007$ \\
\hline & ADMIRE 4 & & $3.8 \pm 0.4$ & $3.9 \pm 0.2$ & $3.4 \pm 0.7^{\mathrm{b}}$ & & \\
\hline \multirow[t]{4}{*}{ Small vessels } & FBP & $3.5 \pm 0.7$ & $2.3 \pm 0.5$ & $2.0 \pm 0.5$ & $1.3 \pm 0.4^{\mathrm{a}, \mathrm{b}}$ & $\mathrm{FD}_{\mathrm{ADM} 2}$ vs. Sn64 ${ }_{\mathrm{ADM} 4}$ & $P=0.468$ \\
\hline & ADMIRE 2 & $3.4 \pm 0.6$ & $3.0 \pm 0.5$ & $2.7 \pm 0.5$ & $2.0 \pm 0.7^{\mathrm{a}, \mathrm{b}}$ & $\mathrm{FD}_{\mathrm{ADM} 2}$ vs. $\mathrm{Sn} 32_{\mathrm{ADM} 4}$ & $P<0.001$ \\
\hline & ADMIRE 3 & & $3.1 \pm 0.6$ & $3.1 \pm 0.4$ & $2.1 \pm 0.4^{\mathrm{a}, \mathrm{b}}$ & $\mathrm{Sn} 64_{\mathrm{ADM} 4}$ vs. Sn32 $2_{\mathrm{ADM} 4}$ & $P=0.028$ \\
\hline & ADMIRE 4 & & $3.2 \pm 0.6$ & $3.1 \pm 0.4$ & $2.5 \pm 0.6^{\mathrm{a}, \mathrm{b}}$ & & \\
\hline \multirow[t]{4}{*}{ Tertiary bronchi } & FBP & $3.8 \pm 0.5$ & $3.1 \pm 0.6$ & $2.8 \pm 0.6$ & $2.2 \pm 0.5^{\mathrm{a}, \mathrm{b}}$ & & \\
\hline & ADMIRE 2 & $3.8 \pm 0.4$ & $3.6 \pm 0.5$ & $3.5 \pm 0.5$ & $3.2 \pm 0.7$ & $\mathrm{FD}_{\mathrm{ADM} 2}$ vs. $\mathrm{Sn} 64_{\mathrm{ADM} 4}$ & ANOVA \\
\hline & ADMIRE 3 & & $3.8 \pm 0.4$ & $3.8 \pm 0.4$ & $3.3 \pm 0.7^{\mathrm{a}, \mathrm{b}}$ & vs. $\mathrm{Sn} 32_{\mathrm{ADM} 4}:$ & $P=0.132$ \\
\hline & ADMIRE 4 & & $3.8 \pm 0.4$ & $3.7 \pm 0.4$ & $3.5 \pm 0.5$ & & \\
\hline \multirow[t]{4}{*}{ Lung fissures } & FBP & $3.3 \pm 0.8$ & $2.2 \pm 0.6$ & $2.2 \pm 0.5$ & $1.3 \pm 0.5^{\mathrm{a}, \mathrm{b}}$ & $\mathrm{FD}_{\mathrm{ADM} 2}$ vs. $\mathrm{Sn} 64_{\mathrm{ADM} 4}$ & $P=0.087$ \\
\hline & ADMIRE 2 & $3.5 \pm 0.7$ & $2.7 \pm 0.7$ & $2.6 \pm 0.8$ & $1.8 \pm 0.8^{\mathrm{a}, \mathrm{b}}$ & $\mathrm{FD}_{\mathrm{ADM} 2}$ vs. $\mathrm{Sn} 32_{\mathrm{ADM} 4}:$ & $P<0.001$ \\
\hline & ADMIRE 3 & & $2.8 \pm 0.8$ & $2.8 \pm 0.6$ & $2.0 \pm 0.6^{\mathrm{a}, \mathrm{b}}$ & $\mathrm{Sn} 64_{\mathrm{ADM} 4}$ vs. Sn32 $2_{\mathrm{ADM} 4}:$ & $P=0.008$ \\
\hline & ADMIRE 4 & & $2.8 \pm 0.7$ & $3.0 \pm 0.6$ & $2.2 \pm 0.5^{\mathrm{b}}$ & & \\
\hline \multirow[t]{4}{*}{ Lung parenchyma } & FBP & $3.5 \pm 0.6$ & $2.0 \pm 0.7$ & $1.6 \pm 0.5^{\mathrm{b}}$ & $1.6 \pm 0.5^{\mathrm{a}}$ & $\mathrm{FD}_{\mathrm{ADM} 2}$ vs. $\mathrm{Sn} 64_{\mathrm{ADM} 4}$ & $P=0.001$ \\
\hline & ADMIRE 2 & $3.6 \pm 0.5$ & $2.7 \pm 0.5$ & $2.6 \pm 0.5$ & $1.8 \pm 0.4^{\mathrm{a}, \mathrm{b}}$ & $\mathrm{FD}_{\mathrm{ADM} 2}$ vs. $\mathrm{Sn} 32_{\mathrm{ADM} 4}$ & $P<0.001$ \\
\hline & ADMIRE 3 & & $2.6 \pm 0.5$ & $2.5 \pm 0.5$ & $2.1 \pm 0.4^{\mathrm{a}}$ & $\mathrm{Sn} 64_{\mathrm{ADM} 4}$ vs. Sn32 $2_{\mathrm{ADM} 4}$ & $P=1$ \\
\hline & ADMIRE 4 & & $2.9 \pm 0.5$ & $2.9 \pm 0.4$ & $2.7 \pm 0.5$ & & \\
\hline
\end{tabular}

Ratings on a 4-point Likert scale ( $1=$ unacceptable, $2=$ acceptable under limited conditions, $3=$ probably acceptable, $4=$ fully acceptable $)$ of the different dose groups. Values are given as mean \pm standard deviation

FBP filtered back-projection, $F D$ full dose, $S n 96 / S n 64 / S n 32$ dose groups with tin prefiltration and different reference tube current time products (96/64/ 32 reference $\mathrm{mAs}$, respectively)

${ }^{\text {a }}$ Values significantly lower compared to Sn96

${ }^{\mathrm{b}}$ Values significantly lower compared to Sn64

${ }^{\mathrm{c}}$ Relevant $P$-values in respect of the hypothesis 
parenchyma, no significant differences in detectability of anatomical structures were found between $\mathrm{FD}_{\mathrm{AM} 2}$ and Sn64 ADM4. On the other hand, differences between Sn64 $4_{\mathrm{ADM} 4}$ and Sn32 2 ADM4 were statistically significant with the exception of tertiary bronchi and lung parenchyma. More detailed information regarding all evaluated anatomical structures and corresponding values of significance is described in Table 2. An example is given in Fig. 1. The two readers disagreed in 281 of 1,120 ratings ( $25 \%$, IBMD 0.10 , 95\% CI 0.08-0.12).

\section{Suspicious lung lesions}

A total of 231 lung lesions were identified on the CT examinations. Mean diameters of the lesions were 6.1 $\pm 5.6 \mathrm{~mm}$ in the full-dose, $5.0 \pm 4.5 \mathrm{~mm}$ in the $\mathrm{Sn} 96,6.2$ $\pm 5.9 \mathrm{~mm}$ in the $\mathrm{Sn} 64$, and $7.6 \pm 6.6 \mathrm{~mm}$ in the $\mathrm{Sn} 32$ groups (ANOVA: $P=0.095$; Table 3 ). The lesions comprised subpleural, peribronchovascular or centrilobular nodules, mucoid impaction, tree-in-bud opacities, septal thickening, local ground-glass opacity, circumscribed consolidations, abscess formation, bronchiectasis, pneumatoceles and cavitations.

Concerning detectability, contrast and contour sharpness of lesions, differences between the $\mathrm{FD}_{\mathrm{ADM}} 2$ group and all $\mathrm{Sn}_{\mathrm{ADM} 4}$ groups turned out to be significant in terms of statistics $(P<0.001$; Table 3$)$. Nevertheless, a Likert score value clearly $>3$ was reached in the $\mathrm{Sn} 96_{\mathrm{ADM}} 4$ and Sn64 $4_{\mathrm{ADM}} 4$ groups regarding lesion detectability (3.4 and 3.3; Fig. 2). Moreover, Sn64 ADM4 only marginally missed a score value of 3 points concerning contrast (2.9) and contour sharpness (2.8). Compared to the Sn64 $4_{\mathrm{ADM}} 4$ group, there were lower score values in the $\mathrm{Sn} 32_{\mathrm{ADM}} 4$ group, being significant concerning contour sharpness $(2.3, P<0.001$; Table 3$)$. An example is given in Fig. 3. The two readers disagreed in 648 of 2,454 ratings $(26 \%$, IBMD $0.12,95 \%$ CI $0.10-0.13)$.

\section{Image quality}

Regarding tin prefiltration, lowering of the reference $\mathrm{mAs}$ effected an increase of noise. With increasing strength levels of ADMIRE, noise significantly decreased in all study groups (Fig. 4). Corresponding values for FBP and ADMIRE 2, 3 and 4 were $78.3 \pm 12.1 \mathrm{HU}, 61.3 \pm 10.5 \mathrm{HU}, 50.3 \pm 9.4 \mathrm{HU}$ and 40.6 $\pm 8.0 \mathrm{HU}$ in the Sn96 group; $92.3 \pm 18.5 \mathrm{HU}, 67.3 \pm 12.9 \mathrm{HU}$, $57.1 \pm 11.6 \mathrm{HU}$ and $45.7 \pm 9.3 \mathrm{HU}$ in the Sn64 group; and 120.9 $\pm 15.4 \mathrm{HU}, 90.2 \pm 12.7 \mathrm{HU}, 75.7 \pm 11.0 \mathrm{HU}$ and $61.5 \pm 9.3 \mathrm{HU}$ in the Sn32 group $(P<0.001)$. An example is given in Fig. 5. Noise value of the Sn64 ${ }_{\mathrm{ADM}} 4$ group did not statistically differ from that in the $\mathrm{FD}_{\mathrm{ADM} 2}$ group ( 45.7 vs. $38.8 \mathrm{HU}, P=0.132$; Fig. 4). On the other hand, noise was significantly higher in the $\mathrm{Sn} 32_{\mathrm{ADM} 4}$ group compared to the $\mathrm{FD}_{\mathrm{ADM} 2}$ group (61.5 vs. $38.8 \mathrm{HU} ; P<0.001)$ and even to the $\mathrm{Sn} 64_{\mathrm{ADM} 4}$ group (61.5 vs. $45.7 \mathrm{HU} ; P<0.001)$.

\section{Radiation exposure and effective dose}

Compared to the full-dose group, the use of tin prefiltration led to a significantly lower radiation exposure

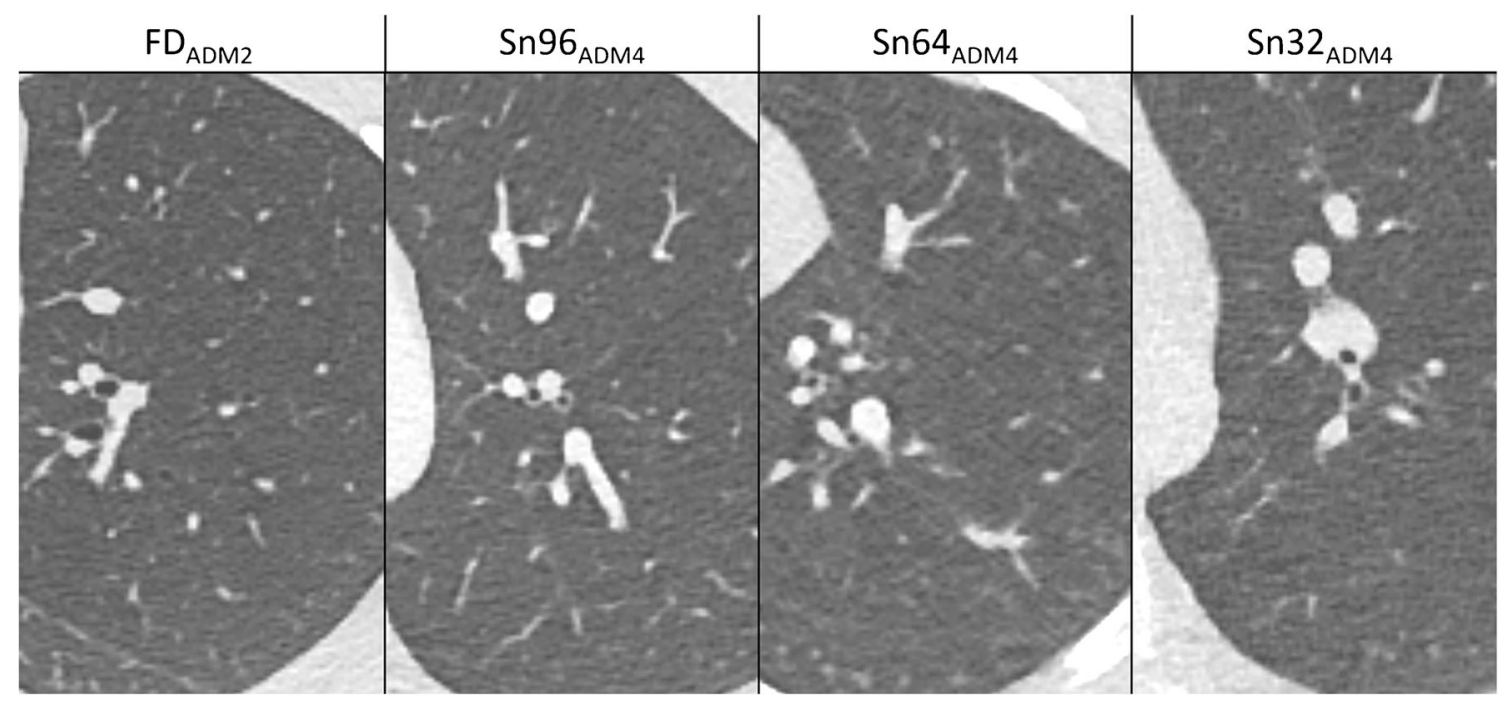

Fig. 1 Examples of comparative detectability of anatomical structures in non-affected lung regions on axial CT slices. FD $_{\text {ADM2 }}$ : full-dose, reconstruction with ADMIRE 2 in a 16-year-old girl with a prolonged course of pneumonia. $\mathbf{S n 9 6}$ ADM $4_{\text {: }}$ tin prefiltration, 96 reference $\mathrm{mAs}$, ADMIRE 4 in a 12-year-old boy with atelectasis of the right upper lobe of unclear origin. $\mathbf{S n 6 4} \mathbf{A D M}_{\mathbf{4}}$ : tin prefiltration, 64 reference $\mathrm{mAs}$, ADMIRE 4 in a 15 -year-old girl with suspected fungal pneumonia.
Sn32 ADM4 $_{\text {: }}$ tin prefiltration, 32 reference mAs, ADMIRE 4 in a 14year-old boy with prolonged course of pneumonia. The detectability of small anatomical structures is acceptable in the $\mathrm{FD}_{\mathrm{ADM} 2}, \mathrm{Sn} 96_{\mathrm{ADM} 4}$ and $\mathrm{Sn} 64_{\mathrm{ADM}} 4$ groups but is limited in the $\mathrm{Sn} 32_{\mathrm{ADM} 4}$ group. $F D$ full-dose group, $\operatorname{Sn} 96 / \mathrm{Sn} 64 / \mathrm{Sn} 32$ groups with tin prefiltration at different reconstruction algorithms (filtered back-projection/ADMIRE 2/3/4) 
Table 3 Characteristics and evaluation of suspicious lung lesions of different dose groups

\begin{tabular}{|c|c|c|c|c|c|}
\hline Dose group & $\mathrm{FD}_{\mathrm{ADM} 2}$ & $\mathrm{Sn} 96_{\mathrm{ADM}}$ & Sn64 ADM4 & $\mathrm{Sn} 32_{\mathrm{ADM}} 4$ & $P$-value $^{\mathrm{a}}$ \\
\hline $\begin{array}{l}\text { Number of } \\
\text { lesions }\end{array}$ & 53 & 61 & 64 & 53 & \\
\hline $\begin{array}{l}\text { Lesions per } \\
\text { patient }\end{array}$ & 3.3 & 3.8 & 4.0 & 3.3 & \\
\hline Size (mm) & $6.1 \pm 5.6$ & $5.0 \pm 4.5$ & $6.2 \pm 5.9$ & $7.6 \pm 6.6$ & $\begin{array}{l}\text { FD vs. Sn96 vs. Sn64 vs. Sn32: } \\
\text { ANOVA } P=0.095\end{array}$ \\
\hline Detectability & $3.8 \pm 0.3$ & $3.4 \pm 0.6$ & $3.3 \pm 0.7$ & $3.0 \pm 0.7$ & $\begin{array}{l}\text { FD vs. } S n 96 / \operatorname{Sn} 64 / \operatorname{Sn} 32: P<0.001 \\
\text { Sn96 vs. } S n 64: P=0.985 \\
\text { Sn64 vs. } S n 32: P=0.125 \\
\text { Sn96 vs. } S n 32: P=0.020\end{array}$ \\
\hline Contrast & $3.6 \pm 0.5$ & $2.9 \pm 0.7$ & $2.9 \pm 0.6$ & $2.6 \pm 0.7$ & $\begin{array}{l}\text { FD vs. } \operatorname{Sn} 96 / \operatorname{Sn} 64 / \operatorname{Sn} 32: P<0.001 \\
\text { Sn96 vs. } S n 64: P=1 \\
\text { Sn64 vs. } S n 32: P=0.177 \\
\text { Sn96 vs. } S n 32: P=0.345\end{array}$ \\
\hline $\begin{array}{l}\text { Contour } \\
\text { sharpness }\end{array}$ & $3.5 \pm 0.6$ & $2.9 \pm 0.8$ & $2.8 \pm 0.7$ & $2.3 \pm 0.7$ & $\begin{array}{l}\text { FD vs. } \mathrm{Sn} 96 / \mathrm{Sn} 64 / \mathrm{Sn} 32: P<0.001 \\
\text { Sn96 vs. } \mathrm{Sn64:} P=0.945 \\
\text { Sn96/Sn64 vs. } \mathrm{Sn} 32: P<0.001\end{array}$ \\
\hline
\end{tabular}

Detectability, contrast and contour sharpness of lesions are rated on a 4-point Likert scale $(1=$ unacceptable, $2=$ acceptable under limited conditions, 3 = probably acceptable, 4 = fully acceptable)

ANOVA analysis of variance, $F D$ full dose, $F D_{A D M 2}$ full dose, reconstruction with ADMIRE 2, Sn $96_{A D M 4}$ $S n 64_{A D M 4} / S n 32_{A D M 4}$ tin prefiltration with 96/64/32 reference mAs, respectively, reconstruction with ADMIRE 4

${ }^{\text {a }}$ Post-hoc pairwise comparisons are displayed when ANOVA $P<0.05$ in the Sn96, Sn64 and Sn32 groups. Mean $\mathrm{CTDI}_{\mathrm{vol}}$ was $2.17 \pm 1.2 \mathrm{mGy}$ vs. $0.31 \pm 0.14$ in the $\mathrm{Sn} 96$ group, 0.24 $\pm 0.10 \mathrm{mGy}$ in Sn64, and $0.13 \pm 0.09 \mathrm{mGy}$ in Sn32 (full dose vs. $\left.\mathrm{Sn}_{\text {all }}: P<0.001\right)$. Corresponding mean DLP was $70.3 \pm 44.0 \mathrm{mGy} \cdot \mathrm{cm}$ in the FD group vs. $10.5 \pm 5.7 \mathrm{mGy} \cdot \mathrm{cm}$ in the Sn96 group, $8.2 \pm 3.4 \mathrm{mGy} \cdot \mathrm{cm}$ in $\mathrm{Sn} 64$, and 3.9 $\pm 2.7 \mathrm{mGy} \cdot \mathrm{cm}$ in $\mathrm{Sn} 32(P<0.001)$. Consequently, this led to a mean effective dose (ED) of $1.26 \pm 0.54 \mathrm{mSv}$ in the FD group vs. $0.21 \pm 0.07 \mathrm{mSv}$ in the $\mathrm{Sn} 96$ group, 0.14 $\pm 0.05 \mathrm{mSv}$ in $\mathrm{Sn} 64$, and $0.07 \pm 0.04 \mathrm{mSv}$ in $\mathrm{Sn} 32$ $(P<0.001)$. Among others, dose reduction was statistically significant between full-dose and Sn64 groups, as well as between $\mathrm{Sn} 64$ and Sn32 groups $(P<0.001)$. Effective dose was reduced to $16.7 \%, 11.1 \%$ and $5.5 \%$ in the Sn96, Sn64 and $\mathrm{Sn} 32$ groups, respectively, compared to the full-dose group (Table 4).

\section{Discussion}

In our retrospective study, pediatric lung dual-source CT examinations with spectral shaping led to significantly lower radiation exposure compared to a full-dose protocol. In terms of statistics, dose lowering to about $10 \%$ by using the Sn64 protocol caused reduction in diagnostic confidence. Nevertheless, acceptable Likert score values $>3$ were achieved for diagnostic confidence as well as detectability of lung lesions when ADMIRE 4 was performed. Simultaneously, there
Fig. 2 Detectability of suspicious lung lesions $(n)$ in the different dose groups rated on a 4-point Likert scale. $F B P$ filtered backprojection, $F D$ full-dose group, Sn96/Sn64/Sn32 groups with tin prefiltration at different reconstruction algorithms (FBP/ADMIRE 2/3/4)

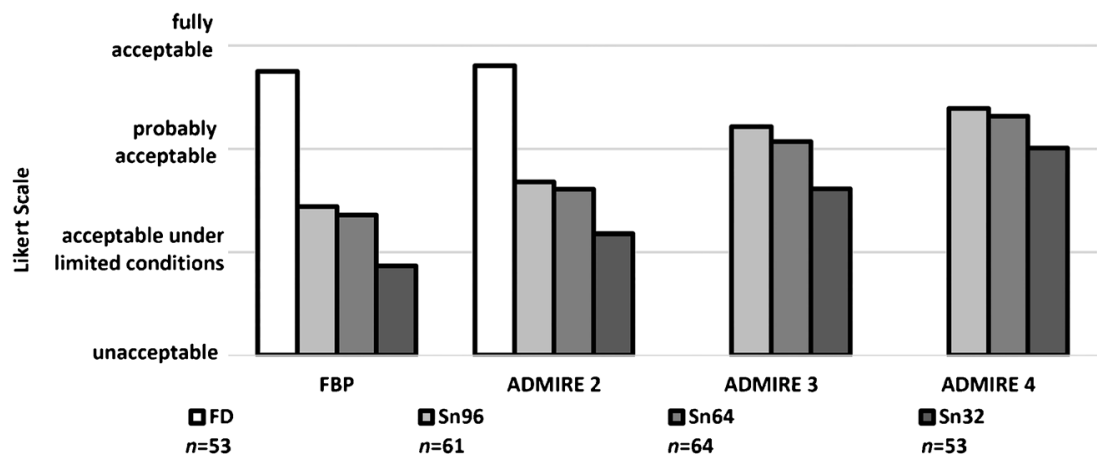




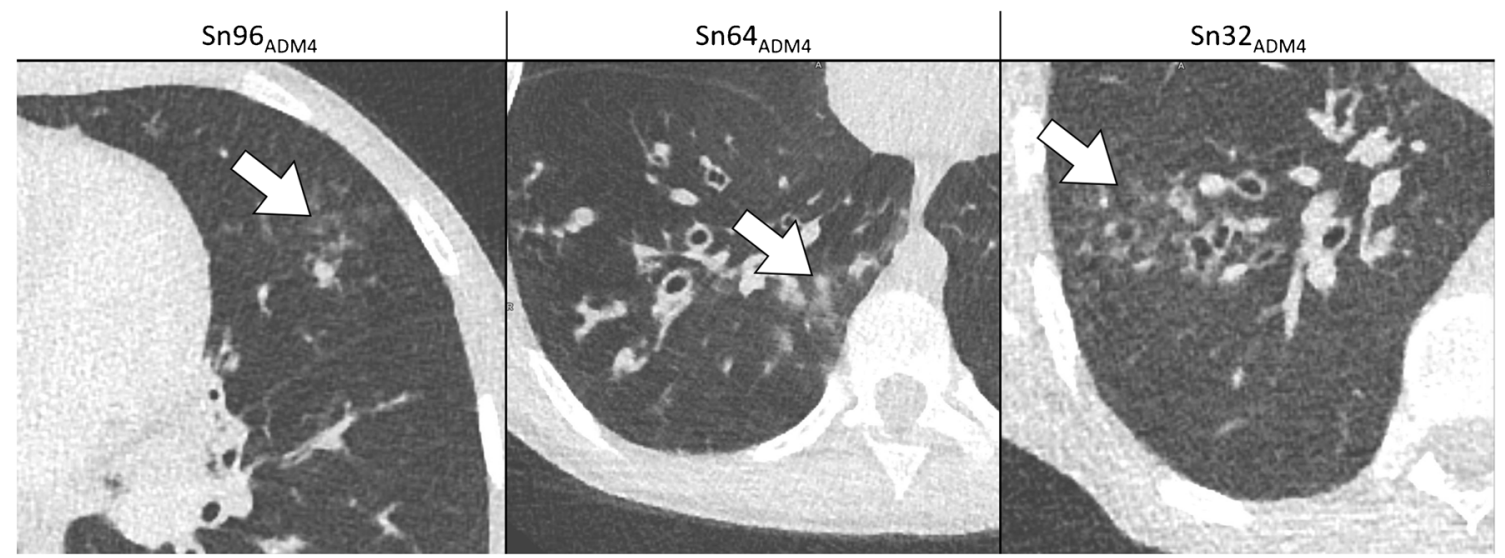

Fig. 3 Examples of comparative detectability of circumscribed consolidations (arrows) of cystic fibrosis on axial CT slices. Sn96 $_{\text {ADM4 }}$ : tin prefiltration, 96 reference mAs, ADMIRE 4 in a 14year-old girl. Sn64 ADM4 $_{\text {: }}$ tin prefiltration, 64 reference mAs, ADMIRE 4 in a 10 -year-old boy. $\mathbf{S n 3 2}$ ADM4: tin prefiltration, 32 reference $\mathrm{mAs}$,

was no significant deterioration of detectability of most anatomical structures, and noise value did not statistically differ from the full-dose group.

There was a significant reduction of radiation exposure between the Sn64 and Sn32 groups. However, further dose reduction to about $5 \%$ of the full-dose group by using the Sn32 protocol caused significant loss of contour sharpness of lung lesions compared to the Sn64 group. Even when ADMIRE 4 was performed, visualization of the majority of anatomical structures was significantly reduced. Diagnostic confidence worsened, and noise significantly increased.

In the last few years, several studies proved the potential of lung CT to deliver adequate image quality when protocols with reduced dose were used [14-16]. In a study by Kroft et al. [15], mean perceived confidence for diagnosis was 98\% for lung CT examinations with a mean effective dose
ADMIRE 4 in a 17-year-old boy. Detectability of circumscribed consolidations is acceptable in the $\mathrm{Sn} 96_{\mathrm{ADM}} 4$ and $\mathrm{Sn} 64_{\mathrm{ADM}} 4$ groups. In the $\mathrm{Sn} 32_{\mathrm{ADM} 4}$ group, interfering noise causes a significant loss of contour sharpness, and detectability is significantly restricted. Sn96/Sn64/Sn32 groups with tin prefiltration at ADMIRE 4 reconstruction algorithm

of $0.07 \mathrm{mSv}$. Ebner et al. [16] investigated chest phantoms with artificial lung nodules between $5 \mathrm{~mm}$ and $12 \mathrm{~mm}$ at a mean dose level of $0.13 \mathrm{mSv}$. Sensitivity for nodule detection was $96.2 \%$ [16]. According to Neroladaki et al. [17], modelbased iterative reconstruction allows secure detection of pulmonary nodules in adults at a radiation dose level of $0.16 \mathrm{mSv}$.

To our knowledge, studies investigating the effect of tin prefiltration on dose reduction are still rare in the pediatric population. Weis et al. [18] compared a $100-\mathrm{kV}$ pediatric chest $\mathrm{CT}$ protocol using spectral shaping $(\mathrm{Sn} 100 \mathrm{kV})$ with a $70-\mathrm{kV}$ standard protocol. Significant dose reduction up to $0.21 \mathrm{mSv}$ and superior subjective image quality of lung structures was achieved with the Sn100-kV protocol. Consequently, their dose results resemble the mean radiation dose of the Sn96 group in

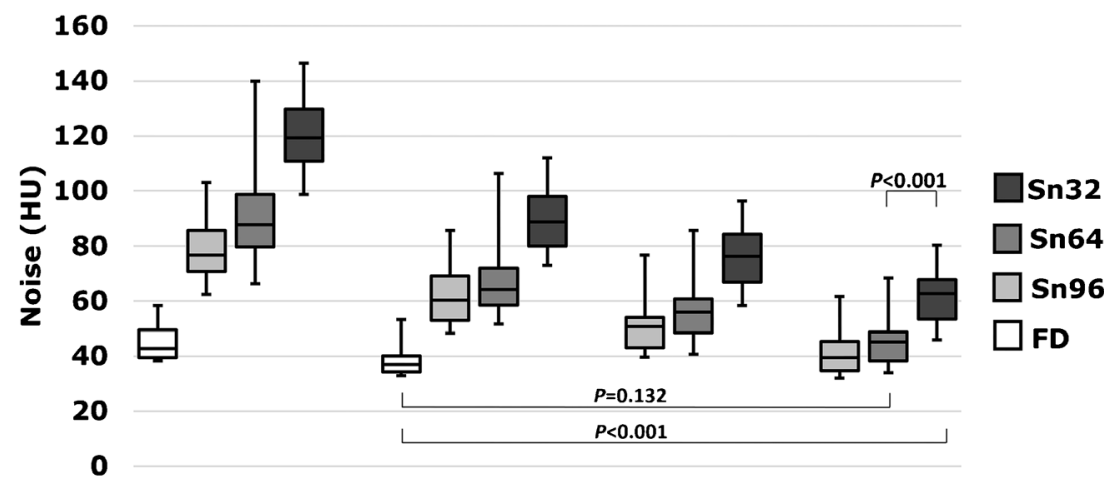

FBP

ADMIRE 2

Fig. 4 Boxplot represents noise measured in the tracheal lumen of patients of the different dose groups. Boxes represent the $25 \%$ and $75 \%$ quartiles, whiskers the minimum and maximum values. Additionally, significance levels of post hoc pairwise comparisons are displayed for $\mathrm{FD}_{\mathrm{ADM} 2}$ vs. Sn64 $4_{\mathrm{ADM} 4} / \mathrm{Sn} 32_{\mathrm{ADM} 4}$ and $\mathrm{Sn} 64_{\mathrm{ADM} 4}$ vs. Sn32 $2_{\mathrm{ADM} 4}$. Noise
ADMIRE 3 ADMIRE 4

did not statistically differ between $\mathrm{FD}_{\mathrm{ADM}}$ group and $\mathrm{Sn}_{6} 4_{\mathrm{ADM}}$ group $(P=0.132)$, whereas noise was significantly higher in the $\mathrm{Sn} 32_{\mathrm{ADM} 4}$ group compared to the $\mathrm{FD}_{\mathrm{ADM} 2} / \mathrm{Sn} 64_{\mathrm{ADM}} 4$ groups $(P<0.001)$. $F D$ fulldose group, $S n 96 / S n 64 / S n 32$ groups with tin prefiltration at different reconstruction algorithms (filtered back-projection/ADMIRE 2/3/4) 


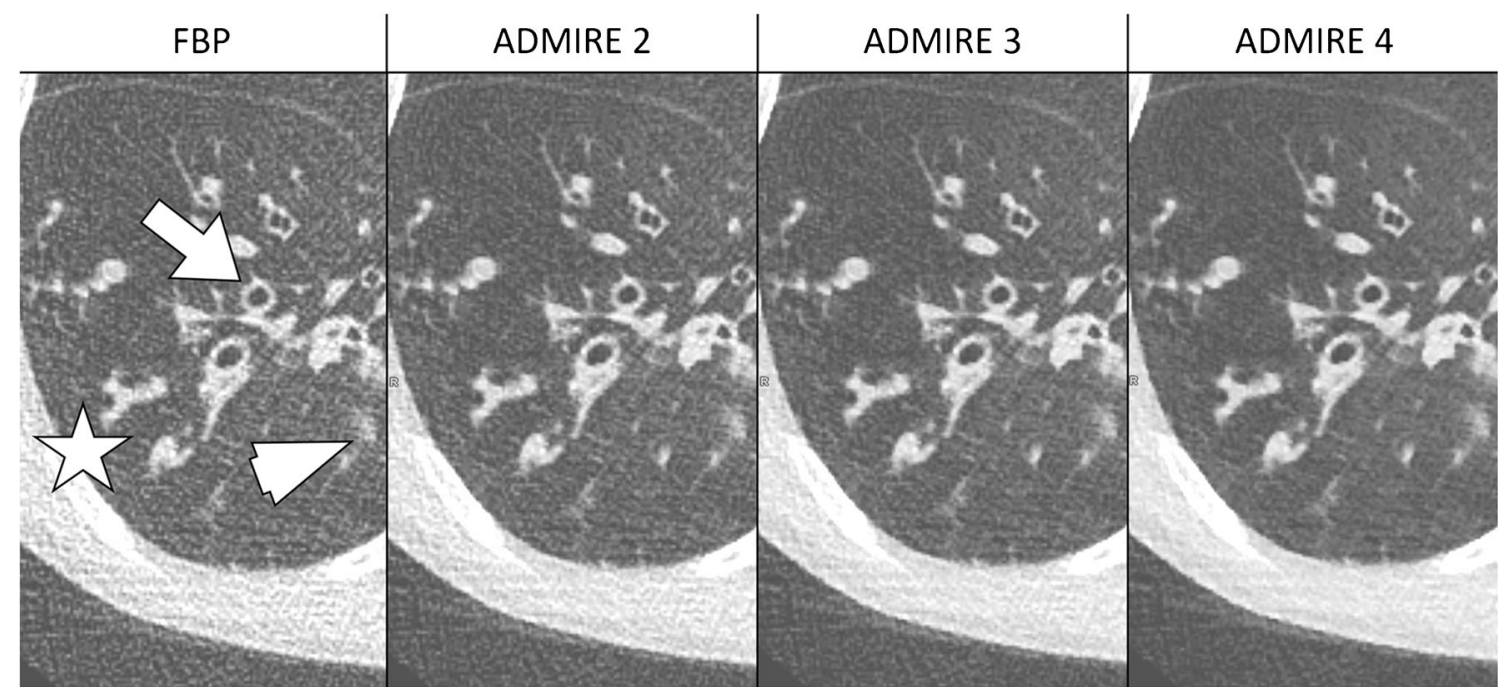

Fig. 5 Influence of reconstruction algorithms (filtered back-projection [FBP], ADMIRE 2/3/4) on image quality and noise in a 10-year-old boy with cystic fibrosis from the Sn64 group (tin prefiltration, 64 reference mAs). Axial CT images depict bronchiectasis (arrow), mucoid impaction (asterisk) as well as circumscribed consolidations (arrowhead). Compared to FBP, noise decreases with increasing strength of ADMIRE our study. In a phantom study, Martini et al. [19] analyzed solid and subsolid lung lesions with low-dose protocols using tin prefiltration. Resulting effective doses were comparable to ours $(0.14 \mathrm{mSv}$ at $1 / 8$ th and $0.05 \mathrm{mSv}$ at $1 / 20$ th of standard dose). They reached diagnostic image quality when using ADMIRE Levels 3 or 5 . Bodelle et al. [5] evaluated the effect of spectral shaping on image quality and effects on radiation parameters using a singlesource $100-\mathrm{kV}$ pediatric chest protocol. With the use of tin prefiltration, increase of effective tube current up to a factor of 10 provided similar image quality with comparable noise at equivalent dose compared to the standard protocol without spectral filtration. Without spectral shaping, CTDI was 3 times higher compared to our Sn96 group, whereas it was still 2.5 times higher when tin prefiltration was added.

This study has some limitations. Because of its retrospective design, patients' age varied from 1.3 years to 18.0 years, with only few small children being included. Therefore our assertions might not be representative for the last-mentioned. Further research is needed in this area, for example with regard to pulmonary metastases in small children with cancer, which was not part of our study. Moreover, we cannot provide sensitivity of lung lesion detection because no internal reference standard was available for comparison. Instead, we evaluated diagnostic confidence and detectability of both anatomical lung structures and suspicious lung lesions. Sensitivity

Table 4 Radiation dose exposure and estimated effective dose among different dose groups

\begin{tabular}{|c|c|c|c|c|c|}
\hline Dose group & FD & Sn96 & Sn64 & $\mathrm{Sn} 32$ & $P$-value ${ }^{\mathrm{a}}$ \\
\hline $\mathrm{CTDI}_{\mathrm{Vol}}$ (mGy) & $2.17 \pm 1.23$ & $0.31 \pm 0.14$ & $0.24 \pm 0.10$ & $0.13 \pm 0.09$ & $\begin{array}{l}\text { FD vs. } S n 96 / \operatorname{Sn} 64 / \mathrm{Sn} 32: P<0.001 \\
\text { Sn64 vs. } S n 32: P=0.008\end{array}$ \\
\hline $\mathrm{DLP}(\mathrm{mGy} \cdot \mathrm{cm})$ & $70.3 \pm 44.0$ & $10.5 \pm 5.7$ & $8.2 \pm 3.4$ & $3.9 \pm 2.7$ & $\begin{array}{l}\text { FD vs. } S n 96 / \mathrm{Sn} 64 / \mathrm{Sn} 32: P<0.001 \\
\text { Sn64 vs. } \mathrm{Sn} 32: P=0.002\end{array}$ \\
\hline $\mathrm{ED}(\mathrm{mSv})$ & $1.26 \pm 0.54$ & $0.21 \pm 0.07$ & $0.14 \pm 0.05$ & $0.07 \pm 0.04$ & $\begin{array}{l}\text { FD vs. } S n 96 / \operatorname{Sn} 64 / \mathrm{Sn} 32: P<0.001 \\
\text { Sn64 vs. Sn32: } P<0.001\end{array}$ \\
\hline $\begin{array}{l}\text { Reduction of ED } \\
\text { (percentage value of FD) }\end{array}$ & & $16.7 \%$ & $11.1 \%$ & $5.5 \%$ & \\
\hline
\end{tabular}

Values of volumetric CT dose index $\left(\mathrm{CTDI}_{\mathrm{Vol}}\right)$, dose-length product (DLP) and effective dose (ED) are given as mean \pm standard deviation FD full-dose group, $\mathrm{Sn} 96 / \mathrm{Sn} 64 / \mathrm{Sn} 32$ groups with tin prefiltration and different reference tube current time products (96/64/32 reference mAs, respectively)

${ }^{a}$ Significant differences $(P<0.05)$ were found between FD group and all Sn groups, but also between Sn64 and Sn32 groups 
regarding detection of small pulmonary lesions with reduced-dose protocols is known to be high. Messerli et al. [20] detected lung nodules in adults with a sensitivity of $91.2 \%$ using a low-radiation-dose protocol comparable to our Sn64 protocol. In a phantom study performed by Grodic et al. [21], sensitivity of pulmonary nodule detection was $94 \%$ in a reduced-dose group with tin prefiltration (1/10th of standard dose) and ADMIRE 5. Although results of sensitivity given from these studies cannot be assigned to our collective, they at least tend to support the validity of our findings.

\section{Conclusion}

In pediatric lung dual-source $\mathrm{CT}$ with spectral shaping, dose reduction to about $10 \%$ of a full-dose protocol still enables acceptable diagnostic quality when image reconstruction is performed with ADMIRE 4.

Acknowledgments Open Access funding provided by Projekt DEAL.

\section{Compliance with ethical standards}

Conflicts of interest Matthias S. May is a member of Siemens Healthcare speakers' bureau. The remaining authors declare that they have no conflicts of interest.

Open Access This article is licensed under a Creative Commons Attribution 4.0 International License, which permits use, sharing, adaptation, distribution and reproduction in any medium or format, as long as you give appropriate credit to the original author(s) and the source, provide a link to the Creative Commons licence, and indicate if changes were made. The images or other third party material in this article are included in the article's Creative Commons licence, unless indicated otherwise in a credit line to the material. If material is not included in the article's Creative Commons licence and your intended use is not permitted by statutory regulation or exceeds the permitted use, you will need to obtain permission directly from the copyright holder. To view a copy of this licence, visit http://creativecommons.org/licenses/by/4.0/.

\section{References}

1. Raff GL (2010) Radiation dose from coronary CT angiography: five years of progress. J Cardiovasc Comput Tomogr 4:365-374

2. May MS, Brand M, Lell MM et al (2017) Radiation dose reduction in parasinus CT by spectral shaping. Neuroradiology 59:169-176

3. Haubenreisser H, Meyer M, Sudarski S et al (2015) Unenhanced third-generation dual-source chest CT using a tin filter for spectral shaping at $100 \mathrm{kVp}$. Eur J Radiol 84:1608-1613

4. Leyendecker P, Faucher V, Labani A et al (2019) Prospective evaluation of ultra-low-dose contrast-enhanced $100-\mathrm{kV}$ abdominal computed tomography with tin filter: effect on radiation dose reduction and image quality with a third-generation dual-source CT system. Eur Radiol 29:2107-2116

5. Bodelle B, Fischbach C, Booz C et al (2017) Single-energy pediatric chest computed tomography with spectral filtration at $100 \mathrm{kVp}$ : effects on radiation parameters and image quality. Pediatr Radiol 47:831-837

6. Vorona GA, Ceschin RC, Clayton BL et al (2011) Reducing abdominal CT radiation dose with the adaptive statistical iterative reconstruction technique in children: a feasibility study. Pediatr Radiol 41:1174-1182

7. Newell JD Jr, Fuld MK, Allmendinger T et al (2015) Very lowdose $(0.15 \mathrm{mGy})$ chest CT protocols using the COPDGene 2 test object and a third-generation dual-source CT scanner with corresponding third-generation iterative reconstruction software. Investig Radiol 50:40-45

8. Rompel O, Glockler M, Janka R et al (2016) Third-generation dualsource $70-\mathrm{kVp}$ chest $\mathrm{CT}$ angiography with advanced iterative reconstruction in young children: image quality and radiation dose reduction. Pediatr Radiol 46:462-472

9. Lell MM, May MS, Brand M et al (2015) Imaging the parasinus region with a third-generation dual-source $\mathrm{CT}$ and the effect of tin filtration on image quality and radiation dose. AJNR Am J Neuroradiol 36:1225-1230

10. Ziegler A, Köhler T, Proksa R (2007) Noise and resolution in images reconstructed with FBP and OSC algorithms for CT. Med Phys 34:585-598

11. Wang G, Yu H, De Man B (2008) An outlook on X-ray CT research and development. Med Phys 35:1051-1064

12. Yu Z, Thibault JB, Bouman CA et al (2011) Fast model-based Xray CT reconstruction using spatially nonhomogeneous ICD optimization. IEEE Trans Image Process 20:161-175

13. Deak PD, Smal Y, Kalender WA (2010) Multisection CT protocols: sex- and age-specific conversion factors used to determine effective dose from dose-length product. Radiology 257:158-166

14. Henriques T, Antunes L, Bernardes J et al (2013) Informationbased measure of disagreement for more than two observers: a useful tool to compare the degree of observer disagreement. BMC Med Res Methodol 13:47

15. Kroft LJM, van der Velden L, Girón IH et al (2019) Added value of ultra-low-dose computed tomography, dose equivalent to chest $\mathrm{X}$ ray radiography, for diagnosing chest pathology. J Thorac Imaging 34:179-186

16. Ebner L, Bütikofer Y, Ott D et al (2015) Lung nodule detection by microdose CT versus chest radiography (standard and dual-energy subtracted). AJR Am J Roentgenol 204:727-735

17. Neroladaki A, Botsikas D, Boudabbous S et al (2013) Computed tomography of the chest with model-based iterative reconstruction using a radiation exposure similar to chest $\mathrm{X}$-ray examination: preliminary observations. Eur Radiol 23:360-366

18. Weis M, Henzler T, Nance JW Jr et al (2017) Radiation dose comparison between $70 \mathrm{kVp}$ and $100 \mathrm{kVp}$ with spectral beam shaping for non-contrast-enhanced pediatric chest computed tomography: a prospective randomized controlled study. Investig Radiol 52:155162

19. Martini K, Higashigaito K, Barth BK et al (2015) Ultralow-dose CT with tin filtration for detection of solid and sub solid pulmonary nodules: a phantom study. Br J Radiol 88:20150389

20. Messerli M, Kluckert T, Knitel M et al (2017) Ultralow dose CT for pulmonary nodule detection with chest X-ray equivalent dose - a prospective intra-individual comparative study. Eur Radiol 27: 3290-3299

21. Grodic S, Morsbach F, Schmidt B et al (2014) Ultralow-dose chest computed tomography for pulmonary nodule detection: first performance evaluation of single energy scanning with spectral shaping. Investig Radiol 49:465-473

Publisher's note Springer Nature remains neutral with regard to jurisdictional claims in published maps and institutional affiliations. 